\title{
Time to lung aeration during a sustained inflation at birth is influenced by gestation in lambs
}

Karen E. McCall ${ }^{1}$, Andreas D. Waldmann ${ }^{2}$, Prue Pereira-Fantini ${ }^{1}$, Regina Oakley ${ }^{1}$, Martijn Miedema ${ }^{1}$, Elizabeth J. Perkins ${ }^{1}$, Peter G. Davis ${ }^{1,3,4}$, Peter A. Dargaville ${ }^{5,6}$, Stephan H. Böhm², Raffaele Dellacà ${ }^{7}$, Magdy Sourial ${ }^{1}$, Emanuela Zannin ${ }^{7}$, Anushi E. Rajapaksa ${ }^{1}$, Andre Tan ${ }^{1}$, Andy Adler ${ }^{8}$, Inéz Frerichs ${ }^{9}$ and David G. Tingay ${ }^{1,3,4,10}$

BACKGROUND: Current sustained lung inflation (SI) approaches use uniform pressures and durations. We hypothesized that gestational-age-related mechanical and developmental differences would affect the time required to achieve optimal lung aeration, and resultant lung volumes, during SI delivery at birth in lambs.

METHODS: 49 lambs, in five cohorts between 118 and 139 days of gestation (term 142 d), received a standardized $40 \mathrm{cmH}_{2} \mathrm{O} \mathrm{Sl}$, which was delivered until $10 \mathrm{~s}$ after lung volume stability (optimal aeration) was visualized on real-time electrical impedance tomography (EIT), or to a maximum duration of $180 \mathrm{~s}$. Time to stable lung aeration $\left(T_{\text {stable }}\right)$ within the whole lung, gravity-dependent, and non-gravitydependent regions, was determined from EIT recordings.

RESULTS: $T_{\text {stable }}$ was inversely related to gestation $(P<0.0001$, Kruskal-Wallis test), with the median (range) being 229 $(85,306) \mathrm{s}$ and $72(50,162) \mathrm{s}$ in the 118-d and 139-d cohorts, respectively. Lung volume at $T_{\text {stable }}$ increased with gestation from a mean (SD) of $20(17) \mathrm{ml} / \mathrm{kg}$ at $118 \mathrm{~d}$ to $56(13) \mathrm{ml} / \mathrm{kg}$ at $139 \mathrm{~d}(P=0.002$, one-way ANOVA). There were no gravitydependent regional differences in $T_{\text {stable }}$ or aeration.

CONCLUSIONS: The trajectory of aeration during an $\mathrm{SI}$ at birth is influenced by gestational age in lambs. An understanding of this may assist in developing SI protocols that optimize lung aeration for all infants.

A $\mathrm{t}$ birth, the fluid-filled fetal lung displays a high resistive state with a prolonged time constant (1-3). Lung aeration immediately after birth relies on the generation of sufficient driving pressure to move lung fluid distally and promote trans-epithelial fluid clearance (3). Preterm infants are often unable to generate the intrathoracic pressures needed to achieve this. A sustained lung inflation (SI), designed to deliver a prolonged positive driving pressure before normal tidal inflation, has been advocated as a potential method of optimizing lung aeration at birth in preterm infants (4). To optimize aeration, both the duration $(2,5)$ and applied pressure of the SI need to be sufficient to overcome the resistance of the lung $(2,3)$. The role of SI pressure has been explored (6), but the factors influencing SI duration are poorly understood. To date, clinical trials of SI have used standardized predetermined fixed durations of 5-15 s, with conflicting results (7-13). This approach assumes a uniform aeration response in all individuals. Our group recently demonstrated that the aeration response of the lung and resultant aeration volume following SI delivery in 127-d gestation preterm lambs were highly variable, although reproducibly predictable, reaching a plateau at five time constants $(1,2,6,14)$. This variability may account for the mixed results of SI in previous studies, and suggests that a uniform SI strategy may expose some recipients to inadequate aeration and others to excessive pressure, both with potentially harmful consequences (15).

Recent advances in electrical impedance tomography (EIT) have enabled monitoring of the aeration response of the lung from birth, allowing individual titration of the SI duration to the aeration response experimentally, with improved gas exchange and lung mechanics compared with a fixed 30-s SI $(2,16)$. Although this approach offers more favorable lung aeration, it is currently a research tool only. Development of safe and effective guidelines for SI application in clinical practice requires exploration of factors that may affect the aeration response of the lung.

The mammalian respiratory system undergoes significant changes during fetal life, and the preterm lung is mechanically and developmentally different from the term lung. Gestational age (GA) may thus affect the aeration response of the lung following SI delivery at birth. We hypothesized that GA differences in the mechanical properties of the lung would significantly influence the time required to reach optimal (stable) lung aeration. Using EIT to individualize SI delivery,

\footnotetext{
${ }^{1}$ Neonatal Research, Murdoch Childrens Research Institute, Parkville, Victoria, Australia; ${ }^{2}$ Swisstom AG, Landquart, Switzerland; ${ }^{3}$ Neonatal Research, The Royal Women's Hospital, Parkville, Victoria, Australia; ${ }^{4}$ Department of Obstetrics and Gynaecology, University of Melbourne, Melbourne, Victoria, Australia; ${ }^{5}$ Department of Paediatrics, Royal Hobart Hospital, Hobart, Tasmania, Australia; ${ }^{6}$ Menzies Institute for Medical Research, University of Tasmania, Hobart, Tasmania, Australia; ${ }^{7}$ TBM Lab, Dipartimento di Elettronica, Informazione e Ingegneria Biomedica-DEIB, Politecnico di Milano University, Milano, Italy; ${ }^{8}$ Department of Systems and Computer Engineering, Carleton University, Ottawa, Ontario, Canada; ${ }^{9}$ Department of Anaesthesiology and Intensive Care Medicine, University Medical Centre Schleswig-Holstein, Campus Kiel, Kiel, Germany; ${ }^{10}$ Department of Neonatology, The Royal Children's Hospital, Parkville, Victoria, Australia. Correspondence: Karen E. McCall (karen.mccall1@nhs.net) 


\section{Role of gestation on aeration at birth Articles}

in five GA cohorts of lambs between a pre-viable developmental state and term, we aimed to determine (i) the relationship between GA and the time to stable lung aeration; (ii) the resultant aeration (lung) volume; and (iii) the parameters that define the exponential temporal aeration relationship of the lung.

\section{METHODS}

The study was performed at the large animal facility of the Murdoch Children's Research Institute (Melbourne, VIC, Australia). The study was approved by our Animal Ethics Committee in accordance with National Health and Medical Research Council (Australia) guidelines.

\section{Fetal Instrumentation}

Lambs of date-mated Border-Leicester cross ewes, raised from the same flock and breeder, were delivered via Cesarean section, under general anesthesia, in five GA cohorts representing the spectrum of gestation-related developmental states from pre-viable to term: 118-120 d (pre-viable), 123-125 d (extreme preterm), 127-129 d (very preterm), 132-134 d (preterm), and 139-141 d (term). All ewes allocated to preterm cohorts received $11.5 \mathrm{mg}$ of betamethasone 24 and $48 \mathrm{~h}$ before delivery. While on placental support the fetal head and neck were exteriorized, the carotid artery and external jugular vein were cannulated, and the trachea was intubated with an appropriately sized cuffed endotracheal tube (ETT) (17). Fetal lung fluid was passively drained ( $15 \mathrm{ml} / \mathrm{kg}$ of estimated birth weight), to emulate conditions consistent with human birth, and the ETT was clamped (2). The thorax was exposed and a 32-electrode EIT belt custom-built for lambs (Swisstom AG, Landquart, Switzerland) was secured around the chest (18). A $10 \mathrm{~s}$ uncalibrated reference EIT recording of the unaerated lung was made to determine fetal (unaerated) values (19), and midazolam, ketamine and propofol sedation was administered intravenously before delivery, to suppress any spontaneous breathing that might initiate lung aeration and bias the results. After delivery, the lambs were weighed, placed supine, and midazolam and ketamine infusions were commenced to suppress spontaneous breathing for the remainder of the study. Physiological measurements were commenced before delivery of the SI. The total time between cord clamping and delivery of the first inflation was less than $60 \mathrm{~s}$ in all lambs.

\section{Sustained Inflation}

On unclamping the ETT, an SI was delivered using a Neopuff T-piece Resuscitator (Fisher Paykel Healthcare, Auckland, New Zealand) at a peak inspiratory pressure of $40 \mathrm{cmH}_{2} \mathrm{O}$, fraction of inspired oxygen $\left(\mathrm{FiO}_{2}\right)$ of 0.3 , and $10 \mathrm{l} / \mathrm{min}$ gas flow. The SI was delivered until $10 \mathrm{~s}$ after volume plateau on the uncalibrated EIT display, or for a maximum of $180 \mathrm{~s}$ (refs 2,16). The lung was held at a positive end-expiratory pressure of $8 \mathrm{cmH}_{2} \mathrm{O}$ for 3-5 s, the ETT was clamped, and the lamb was transferred to an SLE 5000 ventilator (SLE5000, SLE, South Croydon, UK) for ongoing positive pressure ventilation in volume-targeted mode.

\section{Assessment of GA Model}

To determine the mechanical properties and clinical relevance of the GA cohorts, and as part of another study evaluating the role of fetal lung development on expression of early biomarkers of ventilatorinduced lung injury, positive pressure ventilation was continued using a standardized strategy of positive end-expiratory pressure of $8 \mathrm{cmH}_{2} \mathrm{O}$, initial tidal volume of $7 \mathrm{ml} / \mathrm{kg}$, inspiratory time of $0.4 \mathrm{~s}$, maximum peak inspiratory pressure of $40 \mathrm{cmH}_{2} \mathrm{O}$, and a rate of 60 inflations per minute. Continuous monitoring of peripheral oxyhemoglobin saturations $\left(\mathrm{SpO}_{2}\right)$, heart rate, arterial blood pressure, and rectal temperature (HP48S, Hewlett Packard, Andover, MA) ensured lamb's well-being throughout the study period. At $60 \mathrm{~min}$, all lambs were ventilated with $1.0 \mathrm{FiO}_{2}$ for $3 \mathrm{~min}$ and disconnected to atmosphere for $2 \mathrm{~min}$, before an in vivo super syringe pressure-volume measurement was obtained to a maximum pressure of $35 \mathrm{cmH}_{2} \mathrm{O}$ (19). At the end of the study period, lambs received a lethal intravenous dose of pentobarbitone.

Autopsy was performed to obtain histological samples, in order to characterize the developmental state of the lung for each GA cohort. The right upper lobe was fixed at $20 \mathrm{cmH}_{2} \mathrm{O}$ in $4 \%$ paraformaldehyde, and three geographically standardized sites were sampled before further processing of the lung tissue. Histological examination was performed on hematoxylin and eosin-stained formalin-fixed lung sections. Post staining, a minimum of five fields of view (FOV) per site (total $n=15$ FOV) were photographed per lamb (Leica Microsystems, Wetzlar, Germany), and the Image J software (20) was used to determine the percentage of space occupied by airway, alveolar wall thickness, and presence of hyaline membranes. All microscopy assessments were performed by a blinded investigator.

\section{Measurements}

Global and regional thoracic volume changes $\left(\Delta V_{\mathrm{L}}\right)$ were measured continuously from $5 \mathrm{~s}$ before the SI using EIT (Swisstom Pioneer System and STEM software) at 50 frames/s. The EIT $\Delta V_{\mathrm{L}}$ signal was displayed in real-time on a bedside computer monitor (Swisstom STEM software), using an anatomically correct image reconstruction algorithm for the lamb $(18,19)$. Proximal airway pressure $\left(P_{\mathrm{AO}}\right)$ and flow at the airway opening were measured continuously from birth at $200 \mathrm{~Hz}$ using a Florian Respiratory Mechanics monitor (Acutronic AG, Hirzel, Switzerland) (17) in order to calculate dynamic compliance $\left(C_{\mathrm{dyn}}\right)$. Forced oscillation technique measurements were taken for the first $15 \mathrm{~min}$ and immediately after each blood gas analysis by superimposing a $5 \mathrm{~Hz}$ oscillatory pressure onto the ventilation waveform during positive end-expiratory pressure $(2,21)$. Resistance $\left(R_{\mathrm{rs}}\right)$ and reactance $\left(X_{\mathrm{rs}}\right)$ were computed from the forced oscillation technique signals using the method of Dellacà and co-workers $(2,22)$.

\section{Data Analysis}

Global and regional time-course changes in impedance from pre-aeration (immediately before SI) until the end of the SI were reconstructed using the manufacturer's IBEX software package (Swisstom AG), and the heart-beat-related impedance signals were removed as previously described (2). SI duration was determined directly from the time-impedance reconstructed data. The global $\Delta V_{\mathrm{L}}$ from the pre-aerated state was calibrated to $\mathrm{ml} / \mathrm{kg}$ using calibration coefficients derived from the static pressure-volume measurement (2). Relative $\Delta V_{\mathrm{L}}$ within the gravity-dependent and nondependent regions was determined from the raw data. Previous work with our model identified an unequal weighting of lung tissue on computed tomography between the gravity-dependent and nondependent hemithorax permutations on each EIT crosssectional slice, with $63 \%$ of all lung tissue pixels occurring in the dependent hemithorax (19). To account for this, and to determine relative regional volume state, $\Delta V_{\mathrm{L}}$ data were weighted according to the known pixel contribution of each region to global volume, expressed as a fraction of the maximum volume potential in that region (19), with a value of 1.0 suggesting optimal aeration, a value $<1.0$ suggesting lower than expected aeration, and a value $>1.0$ suggesting relative overaeration in that region. Volume states in opposing regions of interest were then compared to determine the homogeneity of the aeration response within the lung.

To allow for the possibility that $\Delta V_{\mathrm{L}}$ had not stabilized within $180 \mathrm{~s}$, and to better describe regional patterns of change, three exponential models (one-phase; plateau followed by one phase; and two phase) were applied to the SI $\Delta V_{\mathrm{L}}$ signals to predict the global and regional time to stable lung volume ( $\left.T_{\text {stable }}\right)$ during each SI, defined as five time constants of the respiratory system $(\tau)(1)$. All models tested identified $R^{2}$ of $0.82-0.99$. As there was no benefit of the more complex models, the simplest one-phase model was selected: 


\section{Articles | Mccall et al.}

$y=y_{0}+\left(y_{\text {plateau }}-y_{0}\right)^{\bullet}(1-\exp (-\mathrm{k} \bullet x)) ; \quad$ where $y_{\text {plateau }}=$ maximum $\Delta V_{\mathrm{L}}, y_{0}=V_{\mathrm{L}}$ immediately before SI, $x=$ time (s), $k=$ reciprocal of $\tau$. $(1,2)$.

\section{Statistical Analysis}

The absolute difference between SI duration across gestations was not known. Previously, in 127-d steroid-exposed lambs of the same species and breeder, a mean (SD) of 78 (31) s was needed to achieve SI plateau (2). A feasibility sample of 10/group would detect a difference of $41 \mathrm{~s}$ between any two groups (power 0.8, alpha error 0.05 ). Data were tested for normality and analyzed using one-way ANOVA or two-way ANOVA, or linear regression as appropriate. All statistical and modeling analyses were performed with GraphPad PRISM 6 (GraphPad Software, SanDiego, CA), and $P<0.05$ was considered significant.

\section{RESULTS}

\section{Fetal Characteristics}

Forty-nine lambs were studied. In seven lambs, data analysis was limited to the SI only because of faulty equipment or incomplete data. As expected, birth weight increased with GA, $P=0.003$, one-way ANOVA (Table 1). Apart from higher drained fetal fluid volumes in the 139 -d cohort $(P=0.002)$, there were no other differences in the fetal characteristics. Eight lambs developed pneumothoraces $(118 \mathrm{~d}, n=4 ; 123 \mathrm{~d}$, $n=4)$ : one at $50 \mathrm{~min}$ of age and the remainder during the static super-syringe pressure-volume (PV) measurement. No other adverse events were noted.

\section{Developmental Differences in the GA Cohorts}

There were clinically predictable GA-related differences in lung mechanics (Figure 1) and histology (Figure 2). The more preterm lambs had lower $X_{\mathrm{rs}}$ and a higher oxygenation index throughout, higher $R_{\mathrm{rs}}$ until $45 \mathrm{~min}$ of age, and poorer $C_{\text {dyn }}$ from $10 \mathrm{~min}$ of age; all had $P$ value $<0.05$ according to Kruskal-Wallis test with Dunn's post tests. The more preterm cohorts had a lower proportion of alveolar spaces $(P<0.0001$, one-way ANOVA), thicker alveolar walls $(P<0.0001)$, and a greater number of hyaline membranes $(P=0.0007)$ than the more mature lambs on histology.

\section{SI Characteristics}

SI duration decreased with increasing GA, $P=0.0001$, Kruskal-Wallis test (Figure 3a). A SI volume plateau was not obtained by $180 \mathrm{~s}$ in $67 \%$ of the 118 -d lambs and $80 \%$ of the 123-d lambs, despite the delivered SI being twice as long compared with the 139-d lambs (median 183 and 183 vs. $94 \mathrm{~s}$ ). Global $\Delta V_{\mathrm{L}}$ increased linearly with GA (Figure $3 \mathrm{~b}$ ), from a mean (SD) of $20(17) \mathrm{ml} / \mathrm{kg}$ at $118 \mathrm{~d}$ to $56(13) \mathrm{ml} / \mathrm{kg}$ at $139 \mathrm{~d}$ (linear regression, $R^{2}=0.96, P=0.003$ ). There were no gestational differences in the relative volume state within the nondependent and dependent regions (Figure 3c), and all gestations had relative heterogeneity of aeration within the lung at the end of the SI (Figure 3d). Like volume state, there was wide variation across gestations, with the highest heterogeneity seen in the 127-d and 139-d cohorts, although neither were significant.

\section{Modeled Time to Lung Volume Stability}

$T_{\text {stable }}$ was inversely related to GA in the global lung, the gravity-dependent, nondependent, left and right regions (Table 2). $T_{\text {stable }}$ was more than three times longer in the 118-d and 123-d cohorts compared with the 139-d (Figure 4a)

Table 1. Gestational age cohort characteristics

\begin{tabular}{|c|c|c|c|c|c|}
\hline & $\begin{array}{l}118-120 \mathrm{~d} \\
\text { (Pre-viable) }\end{array}$ & $\begin{array}{c}\text { 123-125 d (Extreme } \\
\text { preterm) }\end{array}$ & $\begin{array}{l}\text { 127-129 d (Very } \\
\text { preterm) }\end{array}$ & 132-134 d (Preterm) & 139-141 d (Term) \\
\hline$n$ & 9 & 10 & 10 & 9 & 11 \\
\hline Birth weight $(\mathrm{kg})$ & $2.3(0.2)^{*, * *, \dagger}$ & $2.9(0.4)^{* *, \dagger}$ & $3.1(0.7)^{* *, \dagger}$ & $3.9(0.7)$ & $4.5(0.6)$ \\
\hline Fetal lung fluid (ml/kg) & $20.6(4.9)^{* *}$ & $13.4(4.6)^{\dagger}$ & $15.0(6.1)$ & $11.4(7.5)^{\dagger}$ & $21.3(7.0)$ \\
\hline Male $n(\%)$ & $5(56)$ & $6(60)$ & $3(30)$ & 7 (78) & $8(73)$ \\
\hline Twin $n(\%)$ & $8(89)$ & $10(100)$ & $10(100)$ & $9(100)$ & $10(91)$ \\
\hline Cord $\mathrm{pH}$ & $7.29(0.10)$ & $7.33(0.04)$ & $7.32(0.10)$ & $7.32(0.10)$ & $7.29(0.07)$ \\
\hline Cord $\mathrm{PaO}_{2}(\mathrm{mmHg})$ & $23.5(4.4)$ & $24.2(3.2)$ & $24.9(4.2)$ & $21.4(2.1)$ & $22.2(4.3)$ \\
\hline $\begin{array}{l}C_{\text {dyn }}\left(\mathrm{ml} / \mathrm{kg} / \mathrm{cmH}_{2} \mathrm{O}\right) \text { at } \\
60 \mathrm{~min}\end{array}$ & $0.43(0.27,0.48)$ & $0.37(0.27,0.40)^{\ddagger, \S}$ & $0.50(0.39,0.67)$ & $0.55(0.46,0.89)$ & $0.75(0.47,0.92)$ \\
\hline Ol at $60 \mathrm{~min}$ & $19.4(14.8,21.4)^{\ddagger, \S}$ & $20.5(13.5,32.9)^{\ddagger, \S}$ & $8.4(6.2,10.2)$ & $5.5(4.8,6.2)$ & $3.4(2.9,4.0)$ \\
\hline \multicolumn{6}{|l|}{ Histology } \\
\hline Hyaline membranes ( $n$ ) & $7.1(2.6)$ & $8.0(2.4)^{\dagger}$ & $8.7(3.2)^{\dagger}$ & $6.1(1.7)$ & $4.0(1.5)$ \\
\hline
\end{tabular}

$C_{\text {dyn }}$, dynamic compliance; $\mathrm{Ol}$, oxygenation index; $\mathrm{PaO}_{2}$, partial arterial pressure of oxygen.

All data are expressed as mean (SD) except $C_{\text {dyn }}$ and Ol: median (IQR). * vs. 127 d; ${ }^{* *}$ vs. 132 d; $^{\dagger}$ vs. 139 d, $P<0.05$ (one-way ANOVA with Tukey's post tests), ${ }^{\ddagger}$ vs. 132 d; ${ }^{\S}$ vs. $139 \mathrm{~d}, P<0.05$ (Kruskal-Wallis test with Dunn's post tests). 

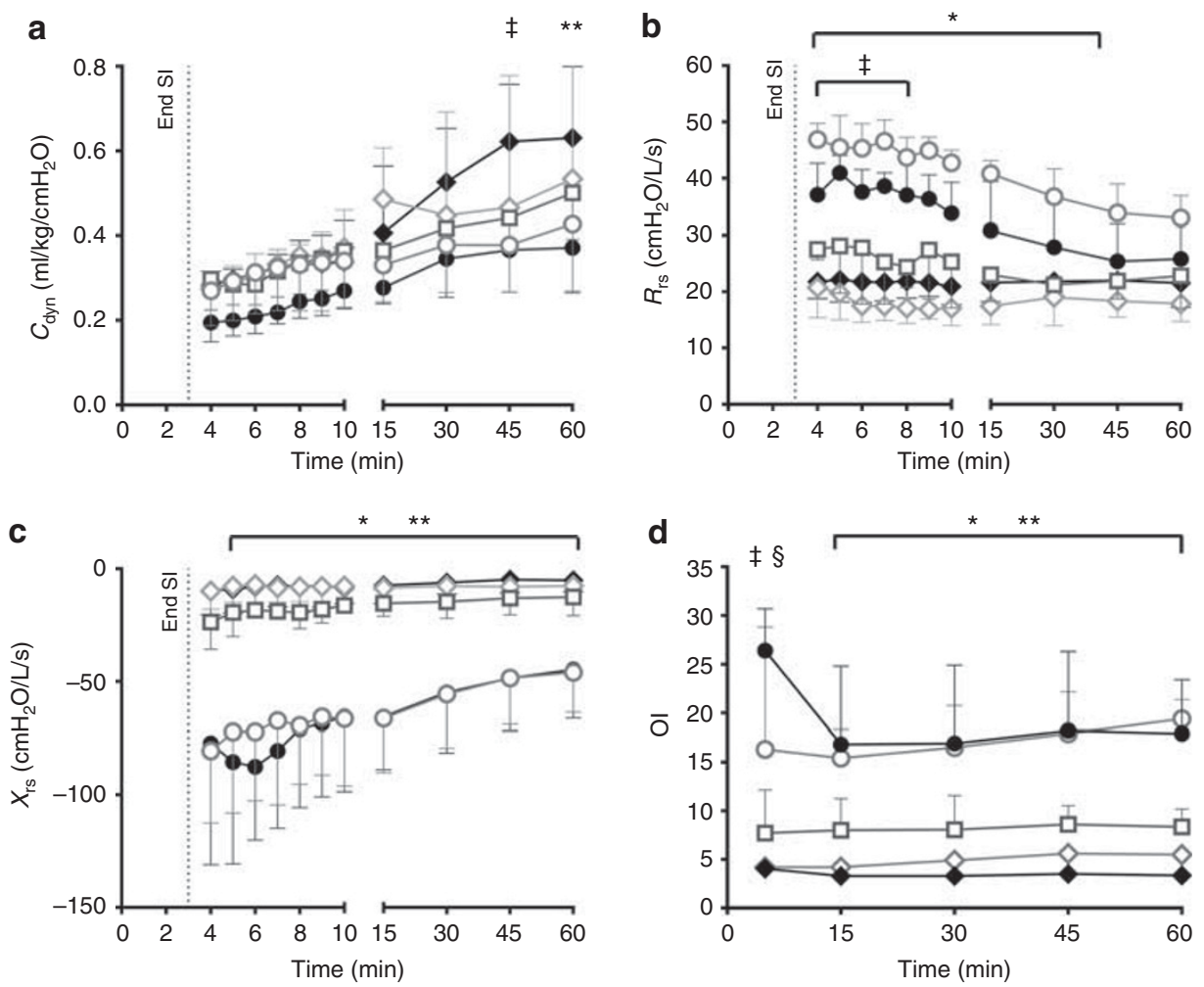

Figure 1. Dynamic compliance $\left(C_{\mathrm{dyn}} ; \mathbf{a}\right)$, resistance $\left(R_{\mathrm{rs}} ; \mathbf{b}\right)$, reactance $\left(X_{\mathrm{rs}} ; \mathbf{c}\right)$, and oxygenation index (Ol; d), respectively, for the 118-d (open circles), 123-d (closed circles), 127-d (squares), 132-d (open diamonds), and 139-d (closed diamonds) cohorts. All data are expressed as median (IQR). $P<0.05$; ${ }^{*} 118 \mathrm{~d}$ vs. 132 and $139 \mathrm{~d},{ }^{* *} 123 \mathrm{~d}$ vs. 132 and $139 \mathrm{~d},{ }^{\dagger} 123 \mathrm{~d}$ vs. $132 \mathrm{~d},{ }^{\ddagger} 123 \mathrm{~d}$ vs. $139 \mathrm{~d},{ }^{\S} 118 \mathrm{~d}$ vs. $139 \mathrm{~d}$ (Kruskal-Wallis with Dunn’s post tests).

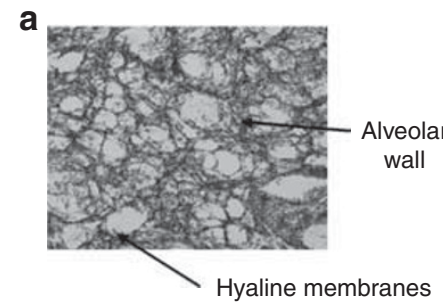

d

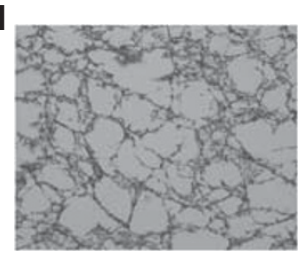

b

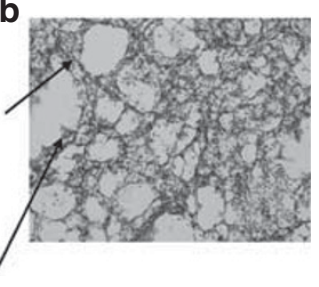

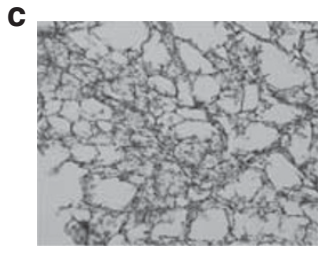

e

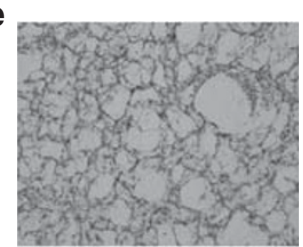

Figure 2. Histological images (H\&E stain) from the right upper lobe for representative 118-d (a), 123-d (b), 127-d (c), 132-d (d), and 139-d lambs (e). All images display the same magnification. The 118-d (pre-viable) image demonstrates lower percentage of alveolar space, thicker alveolar walls, and a greater number of hyaline membranes. H\&E, hematoxylin and eosin.

cohort; median (inter-quartile (IQR)) durations $229(144,261)$ s, $259(191,293) \mathrm{s}$, and $72(66,123) \mathrm{s}$, respectively. The most immature lambs were more likely to have a predicted $T_{\text {stable }}$ significantly longer than $180 \mathrm{~s}$ (Figure $4 \mathbf{b}$ ). Within each GA cohort, gravity-dependent vs. nondependent and left vs. right hemithorax $T_{\text {stable }}$ did not differ (Mann-Whitney test), but values at $118 \mathrm{~d}$ and $123 \mathrm{~d}$ differed from those at more mature gestations (Table 2 and Figure 4c,d).
Temporal Volumetric Behavior of the lung During the SI

Figure 5 shows the complete volumetric behavior of the lung $\left(\Delta V_{\mathrm{L}}\right)$ during the SI for each GA cohort, derived from the reconstructed EIT data and normalized between preaeration (0\%) and end of SI (100\%). The more mature GA cohorts achieved a higher $\Delta V_{\mathrm{L}}$, had a shorter $T_{\text {stable, }}$, and a more rapid rate of initial aeration. Figure 6 summarizes the overall relationship between GA and the volumetric behavior of 

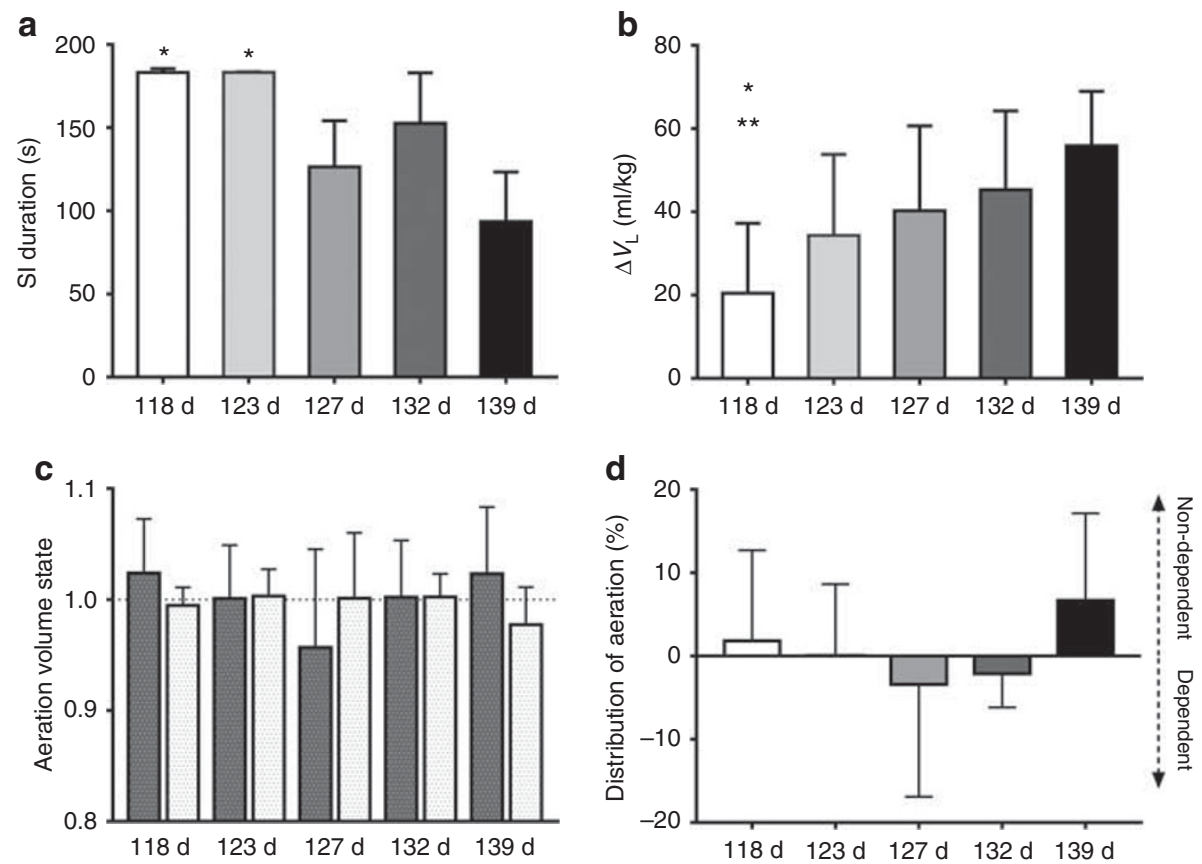

Figure 3. Aeration response of the lung to the delivered SI. (a) Duration of delivered SI. All data are expressed as median (IQR). ${ }^{*}$ vs.139 d, $P<0.05$ (Kruskal-Wallis test with Dunn's post tests). (b) Change in lung volume $\left(\Delta V_{\mathrm{L}}\right)$ at the end of SI from birth. All data are expressed as mean (SD). * vs.139 d; ** vs.132 d, $P<0.05$ (one-way ANOVA with Tukey's post tests). (c) Relative regional aeration volume state in the nondependent (dark gray dotted bar) and dependent (light gray dotted bar) hemithoraces for each GA cohort. Data expressed as a ratio of the actual aeration against expected ventilation in that region for the anatomical contribution of region to total lung tissue EIT pixel count. A value of 1 suggests expected volume state, a value $>1$ suggests overdistension, and a value $<1$ suggests relative atelectasis in that region. All data are expressed as median (IQR). (d) Heterogeneity of gravitational aeration within the EIT cross-sectional slice expressed as the percentage redistribution of aeration (weighted to anatomical contribution) toward the nondependent (positive values) or dependent (negative values) hemithorax. EIT, electrical impedance tomography; GA, gestational age; Sl, sustained lung inflation.

Table 2. Regional time to lung volume stability ( $\left.T_{\text {stable }}\right)$

\begin{tabular}{|c|c|c|c|c|c|c|}
\hline & $\begin{array}{l}118-120 \mathrm{~d} \\
\text { (Pre-viable) }\end{array}$ & $\begin{array}{c}123-125 \mathrm{~d} \text { (Extreme } \\
\text { preterm) }\end{array}$ & $\begin{array}{c}127-129 \mathrm{~d} \text { (Very } \\
\text { preterm) }\end{array}$ & $\begin{array}{l}\text { 132-134 d } \\
\text { (Preterm) }\end{array}$ & $\begin{array}{l}\text { 139-141 d } \\
\text { (Term) }\end{array}$ & $P$ value \\
\hline$T_{\text {stable }}$ Global & $229(144,261)^{5}$ & $259(192,293)^{5}$ & $149(122,188)$ & $142(101,241)$ & $72(66,123)$ & $P<0.0001$ \\
\hline$T_{\text {stable }} \mathrm{L}$ hemithorax & $228(177,280)^{\S}$ & $238(178,283)^{\ddagger, \S}$ & $130(106,163)$ & $92(69,210)$ & $77(65,112)$ & $P=0.0002$ \\
\hline$T_{\text {stable }} \mathrm{R}$ hemithorax & $235(220,278)^{\S}$ & $254(217,254)^{\S}$ & $144(135,207)$ & $133(98,191)$ & $75(63,133)$ & $P<0.0001$ \\
\hline $\begin{array}{l}T_{\text {stable }} \text { non-gravity- } \\
\text { dependent region }\end{array}$ & $224(187,246)^{\S}$ & $258(191,275)^{\S}$ & $124(117,150)$ & $121(89,198)$ & $75(64,122)$ & $P<0.0001$ \\
\hline$P$ value & $P=0.83$ & $P=0.76$ & $P=0.48$ & $P=0.65$ & $P=0.99$ & \\
\hline
\end{tabular}

$T_{\text {stable, time to lung volume stability. }}$

All data are expressed as median $(I \mathrm{QR})^{\ddagger}$ vs. $132 \mathrm{~d}{ }^{\S}{ }^{\S}$ vs. $139 \mathrm{~d}, P<0.05$ (Kruskal-Wallis with Dunn's post tests).

aeration during a single SI at birth. By $60 \mathrm{~s}$, the $139-\mathrm{d}$ cohort had obtained $94 \%$ of final aeration, compared with only 73 and $67 \%$ in the $118-\mathrm{d}$ and $123-\mathrm{d}$ cohorts, respectively, $P<0.0001$, one-way ANOVA with Tukey's post tests. There were no differences in the modeled regional volumetric behavior (Table 2).

\section{DISCUSSION}

Achieving lung aeration at birth is essential to establishing effective ventilation. SIs are increasingly advocated for this purpose, but the influence of the developmental lung state on this therapeutic maneuver is poorly understood. Our preterm lamb study evaluated the role of GA on initial lung aeration using an SI, the first to our knowledge to do so systematically in an appropriate model of the human lung. We found significant differences in the temporal behavior of lung aeration and absolute aeration volume, related to the gestational mechanical and developmental properties of the lung. Increasing prematurity was associated with slower aeration patterns and lower aeration volumes. Although this is an animal study, our findings have important implications for clinical practice. Clinical uses of SI have focused on 


\section{Role of gestation on aeration at birth $\quad$ Articles}
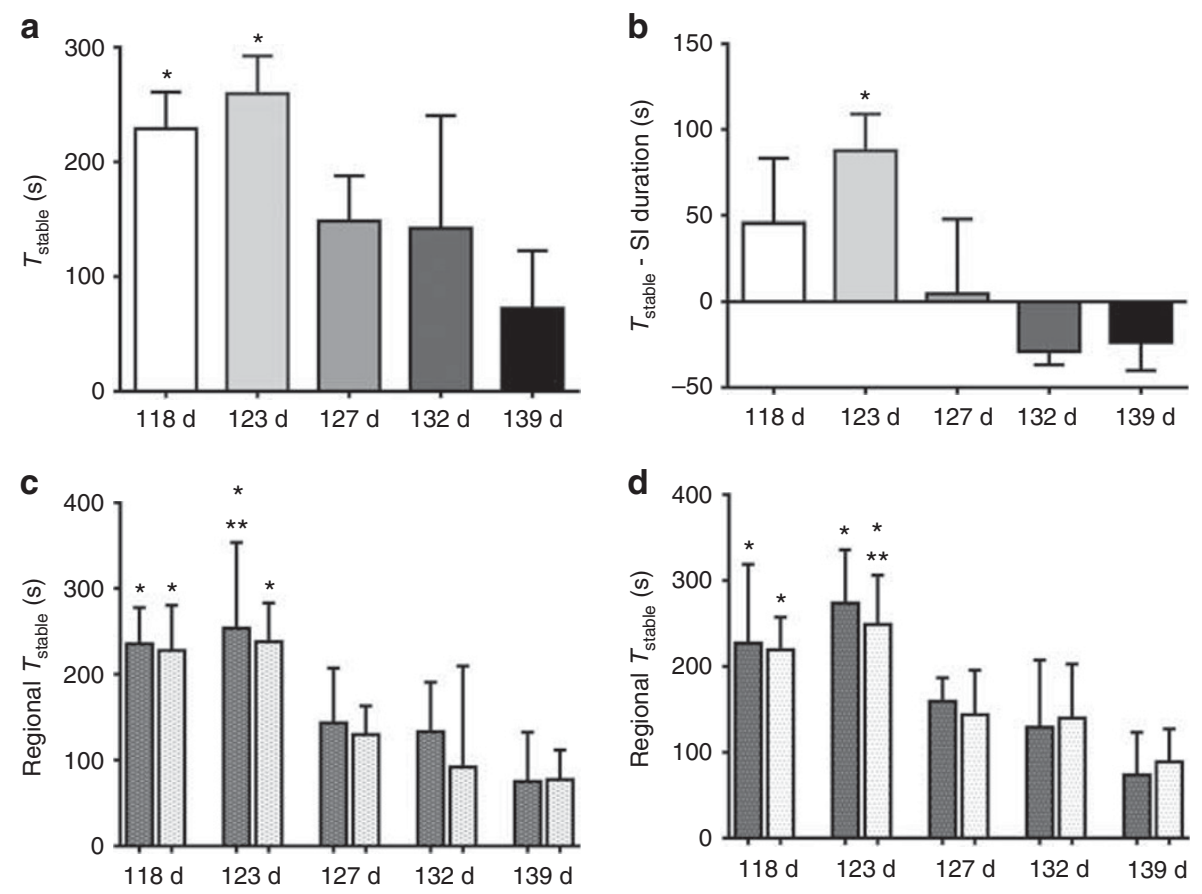

Figure 4. Predicted global and regional time to stable lung volumes ( $T_{\text {stable }}$ ). (a) Predicted $T_{\text {stable }}$ in the whole lung (global) by GA, calculated using a one-phase exponential model $y=y_{0}+\left(y_{\text {plateau }}-y_{0}\right)^{\prime}(1-\exp (-k x))$, where $y_{\text {plateau }}=$ maximum $\Delta V_{\mathrm{L}}, y_{0}=V_{\mathrm{L}}$ immediately before $\mathrm{Sl}, x=$ time $(\mathrm{s})$, $k=$ reciprocal of time constant $(\tau)$. (b) Difference between actual delivered SI duration (maximum $180 \mathrm{~s}$ ) and predicted $T_{\text {stable. }}$ (c) Regional predicted $T_{\text {stable }}$ within the right (dark gray dotted bar) and left (light gray dotted bar) hemithoraces and (d) nondependent (dark gray dotted bar) and dependent (light gray dotted bar) hemithoraces by GA. All data are expressed as median (IQR). p <0.05: * vs.139 d; ** vs.132 d; (Kruskal-Wallis test with Dunn's post tests). GA, gestational age; $\mathrm{Sl}$, sustained lung inflation; $T_{\text {stable, }}$ time to lung volume stability.
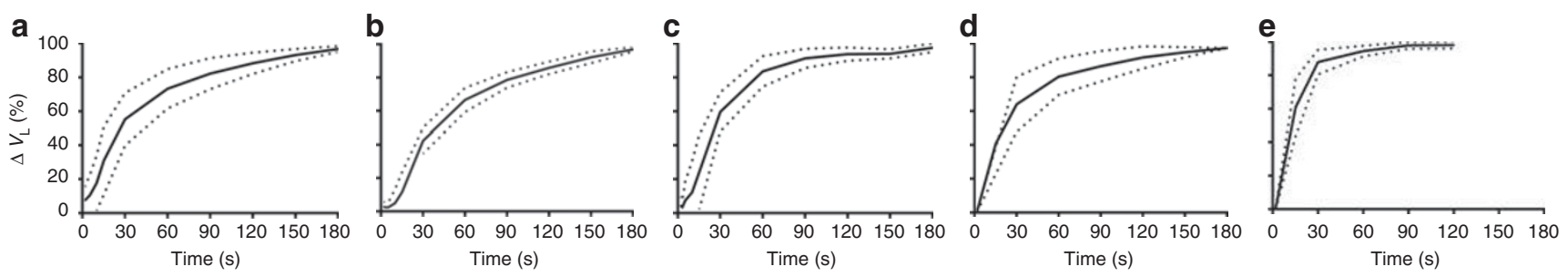

Figure 5. Volumetric behavior of the lung during the SI (maximum duration $180 \mathrm{~s}$ ) normalized in each animal to global $\Delta V_{\mathrm{L}}$. for 118-d (a), 123-d (b), 127-d (c), 132-d (d), and 139-d (e) GA cohorts. Solid line represents mean and dashed lines represent SD. GA, gestational age.

predetermined SI duration and driving pressures, without mandating differences in duration by GA. Such approaches are unlikely to optimize lung aeration within all individuals, and may create injurious conditions, especially if combined with inappropriate driving pressures.

Fundamental to the respiratory transition at birth is the need to apply sufficient hydrostatic driving pressure to move lung liquid from airways and alveoli into the interstitium, thus allowing alveolar aeration and establishment of FRC before tidal ventilation (3). Because of the long time constants of the fluid-filled fetal lung, and the knowledge that volume change in the lung is determined by the product of pressure and time, an SI has been advocated as a potential method of optimizing lung aeration $(3,4,23)$. However, both animal $(1,6,14,17$, 24-28) and human (7-13) studies have shown conflicting results, often inconsistent with the predicted mechanism of action of an SI (5). A commonality of these studies was the use of pre-determined, and fixed, SI durations. This seems incongruous with the current focus on lung protection, as it does not account for the highly variable mechanical conditions of the diseased infant lung (29-32). Increasingly, clinicians are using individualized ventilation strategies, such as open-lung ventilation and volume-targeting, in an effort to avoid harmful lung volume states and achieve regional homogeneity $(7,32)$. Previous work from our group, limited to a single GA, demonstrated great heterogeneity in the time constants of the respiratory system at birth, which influenced SI aeration outcomes $(2,14,16)$. We have now demonstrated that GA independently influences aeration during an SI.

Preterm infants from as early as 23 weeks of gestation are now routinely actively resuscitated, and they are most likely to benefit from lung protective resuscitation. Prematurity represents the most frequent need for respiratory support at birth. There is increasing awareness that lung injury may 


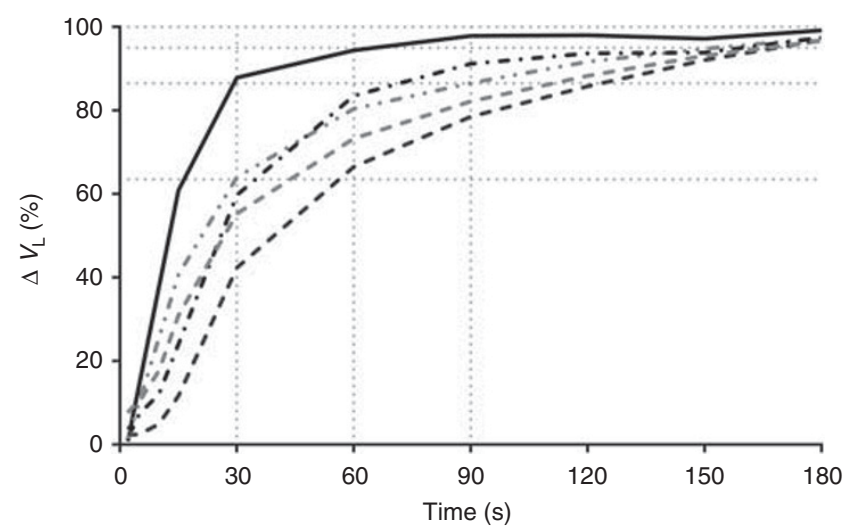

Figure 6. Summary of the relationship between GA and the volumetric behavior of the lung $\left(\Delta V_{\mathrm{L}}\right)$ during an SI as the first inflation at birth for the 118-d (dashed dark gray line), 123-d (dashed black line), 127-d (dot/ dash dark gray line), 132-d (dot/dash black line), and 139-d (black line) GA cohorts. $\Delta V_{\mathrm{L}}$ represents the mean change for that cohort expressed as normalized change from preaeration (0\%) and $\Delta V_{\mathrm{L}}$ at the end of $\mathrm{SI}$ (100\%; maximum volume). Gray dotted lines illustrate $\Delta V_{\mathrm{L}}$ at 30,60 , and $90 \mathrm{~s}(x$ axis) and by time constant $(\operatorname{tau}, \tau)$ at $63.5 \%(\tau), 86.5 \%(2 \tau), 95 \%$ (4T), and $99 \%(5 \tau)$ of maximum $\Delta V_{\mathrm{L}}$ (y axis). The more preterm groups achieved stable $\Delta V_{\mathrm{L}}$ later, with the $118-\mathrm{d}$ cohort achieving $50 \%$ of $139-\mathrm{d}$ cohort $\Delta V_{\mathrm{L}}$ at $30 \mathrm{~s}$. GA, gestational age; $\mathrm{Sl}$, sustained lung inflation.

begin from the first inflation (33), focusing attention on lungprotective strategies in the delivery room. The last 20 weeks of gestation are associated with significant changes in fetal lung size, alveolar morphology, and surfactant function, along with increasing central drive and chest wall maturity $(15,34)$. The finding in our current study that immature gestation was associated with longer respiratory time constants was fully expected. This, along with the histological and mechanical data following the SI, demonstrates that our GA cohorts were sufficiently different and representative of the spectrum of preterm infants in the NICU (35). The majority of lamb studies have used SI durations $\leq 60$ s (refs 1,6,14,17,24-28). Only the term lambs in our study population had achieved stable aeration (five time constants) within $60 \mathrm{~s}$, at the inflating pressure we used. By contrast, $60 \mathrm{~s}$ approximated only one time constant for the $118-\mathrm{d}$ and $123-\mathrm{d}$ groups. The lamb has similar fetal lung development as humans but different chest wall characteristics, and more lung liquid (19). Thus, in absolute terms, the $T_{\text {stable }}$ durations we report are likely to be longer than those needed in human infants. Notwithstanding this, the relative magnitude of the differences in time constants at birth we observed would translate to significant SI duration requirements in humans, more so than currently accounted for in existing clinical trials.

The preterm lung has smaller total lung capacity than the term lung, a finding of our study, and larger relative deadspace because of reduced alveolar volume (15). A corollary of this is that targeting a delivered volume during an SI using airway opening volume, as previously advocated (26), is unlikely to achieve uniform aeration across gestations. Such an approach may result in injurious volume states if the delivered volume is too small or large for the recipient's lung.
Unlike respiratory support in the already aerated infant, child, or adult lung, the mechanical state of the lung and total lung capacity at birth is not known. This highlights the need to target the relative volumetric change rather than an absolute value. Direct feedback of the aeration process will be needed if preterm respiratory failure is to be supported with the same sophistication as in the NICU. Of the current delivery room assessment tools, only expired $\mathrm{CO}_{2}$ allows such an approach (36), but is ETT leak-dependent. The latter is important, as unlike our study most infants are managed at birth with a facemask. Using current anatomically correct image reconstruction EIT algorithms offers direct visualization of aeration and ventilation independent of ETT leak, at least within the examined chest section (37). Although adult and pediatric EIT devices are available for use in the clinical setting, EIT use in neonates is currently limited to research (37). The development of rapid application electrode belts and instantaneously available online analysis during examination $(37,38)$, as used in our study, offers future potential in the delivery room to guide both pressure and duration of SI and other respiratory strategies.

The use of EIT-guided SI delivery via a cuffed ETT resulted in homogeneous distribution of aeration between the gravitydependent and nondependent regions of the lung, indicating similar regional time constants. This differs from studies of preterm infants in the NICU (27) and adults with surfactantdeficient acute respiratory failure (39), which are diseases characterized by gravity-dependent differences secondary to reduced $C_{\text {dyn }}$. The larger contribution of resistance in the fluid-filled fetal lung would explain our differences. Regional aeration volumes were similar to the anatomical potential, suggesting sufficient SI durations to achieve homogeneous aeration. The aeration heterogeneity noted in the 139-d cohort, with nondependent lung overdistension, is likely a consequence of excessive SI duration. This also illustrates the need to monitor the pattern of the time-volume relationship, as rapid change may generate injurious shear forces. Despite different resultant volumes, the time-volume relationship was similar across gestations, and predictable using a simple exponential model. This offers the potential for integration into resuscitation monitors and algorithms.

In our lambs, unlike human infants, spontaneous breathing was suppressed and the SI was delivered via a cuffed ETT to ensure reliable driving pressure delivery and mechanical evaluation. Most human infants have some spontaneous effort, or conversely irregular glottis function, which may alter aeration patterns and the reliability of the modeling. We contend that the influence of development on the mechanical properties of the lung applies irrespective of central respiratory drive and clinical care. The SI inflating pressure and subsequent positive end-expiratory pressure settings were based on values known to optimize outcomes in preterm lambs. It is possible that these values were not optimal for the more mature gestations evaluated $(17,40)$. Despite being larger than most preterm lamb studies, our sample size of 9-11 per group may not have been sufficient to account for 
within-group variability, especially as the ratio of male infants was not constant (although not statistically different) (16). This suggests that future physiological and interventional human studies of aeration at birth will need large sample sizes.

\section{CONCLUSIONS}

In our preterm lamb study, GA exerted a significant, and linear, influence on the volumetric behavior of the lung during an SI, delivered as the first inflation at birth. Longer SI durations were needed to achieve optimal aeration in the most preterm lambs. Clinical strategies at birth that use similar predetermined SI durations across broad gestations are likely to be too simplistic, and may inadvertently generate injurious volume states in some individuals. The highly predictable exponential time-volume behavior of the lung during the first inflation, irrespective of gestation, offers potential for mechanically appropriate individualized SI strategies to be developed.

\section{AUTHOR CONTRIBUTIONS}

K.E.McC., D.G.T., R.D., P.A.D., and P.G.D. developed the concept, designed the experiment, interpreted the data, and wrote the manuscript. K.E.McC. wrote the first draft. P.P., R.O., M.M., E.J.P., M.S., A.R., and A.T. were involved in all lamb experimental work. A.W. and A.A. designed the EIT image reconstruction algorithms. K.E.McC., A.T., and A.W. performed the data analysis. K.E.McC., D.G.T., A.A., M.M., and I.F. interpreted the EIT data. R.D. and E.Z. performed the forced-oscillation technique analysis. All authors contributed to redrafting the manuscript.

\section{STATEMENT OF FINANCIAL SUPPORT}

This study is supported by a National Health and Medical Research Council Project Grant, Canberra, Australia (Grant ID 1009287) and the Victorian Government Operational Infrastructure Support Program (Melbourne, Australia). D.G.T. is supported by a National Health and Medical Research Council Clinical Career Development Fellowship (Grant ID 1053889).

Disclosure: S.B. and A.W. are employees of Swisstom AG, a company producing commercial adult EIT and research devices. Swisstom customized the EIT hardware and software for the preterm lamb model used in this report using open-source software designed by A.A. Both helped interpret the EIT data and revised the manuscript. All EIT hardware and software used in this study were purchased at commercial rates by the Murdoch Childrens Research Institute without restrictions or obligations. The remaining authors declare no conflict of interest.

\section{REFERENCES}

1. Tingay DG, Wallace MJ, Bhatia R, et al. Surfactant before the first inflation at birth improves spatial distribution of ventilation and reduces lung injury in preterm lambs. J Appl Physiol 2014;116:251-8.

2. Tingay DG, Lavizzari A, Zonneveld CE, et al. An individualized approach to sustained inflation duration at birth improves outcomes in newborn preterm lambs. Am J Physiol Lung Cell Mol Physiol 2015;309:L1138-49.

3. Hooper SB, Te Pas AB, Kitchen MJ. Respiratory transition in the newborn: a three-phase process. Arch Dis Child Fetal Neonatal Ed 2016;101:F266-71.

4. McCall KE, Davis PG, Owen LS, Tingay DG. Sustained lung inflation at birth: what do we know, and what do we need to know? Arch Dis Child Fetal Neonatal Ed 2016;101:F175-80.

5. Te Pas AB, Siew M, Wallace MJ, et al. Effect of sustained inflation length on establishing functional residual capacity at birth in ventilated premature rabbits. Pediatr Res 2009;66:295-300.
6. Polglase GR, Tingay DG, Bhatia R, et al. Pressure- versus volume-limited sustained inflations at resuscitation of premature newborn lambs. BMC Pediatr 2014;14:43-52.

7. Harling AE, Beresford MW, Vince GS, Bates M, Yoxall CW. Does sustained lung inflation at resuscitation reduce lung injury in the preterm infant? Arch Dis Child Fetal Neonatal Ed 2005;90:F406-10.

8. Te Pas AB, Walther FJ. A randomized, controlled trial of delivery-room respiratory management in very preterm infants. Pediatrics 2007;120: 322-9.

9. Lista G, Fontana P, Castoldi F, Cavigioli F, Dani C. Does sustained lung inflation at birth improve outcome of preterm infants at risk for respiratory distress syndrome? Neonatology 2011;99:45-50.

10. Lista G, Boni L, Scopesi F, et al. Sustained lung inflation at birth for preterm infants: a randomized clinical trial. Pediatrics 2015;135: e457-64.

11. Grasso C, Sciacca P, Giacchi V, et al. Effects of Sustained Lung Inflation, a lung recruitment maneuver in primary acute respiratory distress syndrome, in respiratory and cerebral outcomes in preterm infants. Early Hum Dev 2015;91:71-5.

12. Lindner W, Vossbeck S, Hummler H, Pohlandt F. Delivery room management of extremely low birth weight infants: spontaneous breathing or intubation? Pediatrics 1999;103:961-7.

13. Lindner W, Hogel J, Pohlandt F. Sustained pressure-controlled inflation or intermittent mandatory ventilation in preterm infants in the delivery room? A randomized, controlled trial on initial respiratory support via nasopharyngeal tube. Acta Paediatr 2005;94:303-9.

14. Tingay DG, Polglase GR, Bhatia R, et al. Pressure-limited sustained inflation versus gradual tidal inflations for resuscitation in preterm lambs. J Appl Physiol 2015;118:890-7.

15. Jobe AH, Hillman N, Polglase G, Kramer BW, Kallapur S, Pillow J. Injury and inflammation from resuscitation of the preterm infant. Neonatology 2008;94:190-6.

16. Tingay DG, Rajapaksa A, Zannin E, et al. Effectiveness of individualized lung recruitment strategies at birth: an experimental study in preterm lambs. Am J Physiol Lung Cell Mol Physiol 2017;312:L32-41.

17. Tingay DG, Bhatia R, Schmolzer GM, Wallace MJ, Zahra VA, Davis PG. Effect of sustained inflation vs. stepwise PEEP strategy at birth on gas exchange and lung mechanics in preterm lambs. Pediatr Res 2014;75: 288-94.

18. Miedema M, McCall KE, Perkins EJ, et al. First real-time visualization of a spontaneous pneumothorax developing in a preterm lamb using electrical impedance tomography. Am J Respir Crit Care Med 2016;194: $116-8$.

19. Tingay DG, Rajapaksa A, Zonneveld CE, et al. Spatiotemporal aeration and lung injury patterns are influenced by the first inflation strategy at birth. Am J Respir Cell Mol Biol 2016;54:263-72.

20. Schneider CA, Rasband WS, Eliceiri KW. NIH Image to ImageJ: 25 years of image analysis. Nat Methods 2012;9:671-5.

21. Dellacà RL, Zannin E, Ventura ML, et al. Assessment of dynamic mechanical properties of the respiratory system during high-frequency oscillatory ventilation. Crit Care Med 2013;41:2502-11.

22. Dellacà RL, Zannin E, Kostic $\mathrm{P}$, et al. Optimisation of positive end-expiratory pressure by forced oscillation technique in a lavage model of acute lung injury. Intensive Care Med 2011;37:1021-30.

23. Schmölzer GM, Kumar M, Aziz K, et al. Sustained inflation versus positive pressure ventilation at birth: a systematic review and meta-analysis. Arch Dis Child Fetal Neonatal Ed 2015;100:F361-8.

24. Te Pas AB, Siew M, Wallace MJ, et al. Establishing functional residual capacity at birth: the effect of sustained inflation and positive endexpiratory pressure in a preterm rabbit model. Pediatr Res 2009;65: $537-41$.

25. Klingenberg C, Dawson JA, Gerber A, Kamlin CO, Davis PG, Morley CJ. Sustained inflations: comparing three neonatal resuscitation devices. Neonatology 2011;100:78-84. 


\section{Articles | Mccall et al.}

26. Sobotka KS, Hooper SB, Allison BJ, et al. An initial sustained inflation improves the respiratory and cardiovascular transition at birth in preterm lambs. Pediatr Res 2011;70:56-60.

27. Sobotka KS, Hooper SB, Crossley KJ, et al. Single sustained inflation followed by ventilation leads to rapid cardiorespiratory recovery but causes cerebral vascular leakage in asphyxiated near-term lambs. PLoS ONE 2016;11:e0146574.

28. Hillman NH, Kemp MW, Noble PB, Kallapur SG, Jobe AH. Sustained inflation at birth did not protect preterm fetal sheep from lung injury. Am J Physiol Lung Cell Mol Physiol 2013;305:L446-53.

29. Miedema M, de Jongh FH, Frerichs I, van Veenendaal MB, van Kaam AH. Regional respiratory time constants during lung recruitment in high-frequency oscillatory ventilated preterm infants. Intensive Care Med 2012;38:294-9.

30. Tingay DG, Mills JF, Morley CJ, Pellicano A, Dargaville PA. The deflation limb of the pressure-volume relationship in infants during highfrequency ventilation. Am J Respir Crit Care Med 2006;173:414-20.

31. Tingay DG, Mills JF, Morley CJ, Pellicano A, Dargaville PA. Indicators of optimal lung volume during high-frequency oscillatory ventilation in infants. Crit Care Med 2013;41:237-44.

32. Pellicano A, Tingay DG, Mills JF, Fasulakis S, Morley CJ, Dargaville PA. Comparison of four methods of lung volume recruitment during high frequency oscillatory ventilation. Intensive Care Med 2009;35:1990-8.

33. Bjorklund LJ, Ingimarsson J, Curstedt T, et al. Manual ventilation with a few large breaths at birth compromises the therapeutic effect of subsequent surfactant replacement in immature lambs. Pediatr Res 1997;42:348-55.

34. Smith LJ, McKay KO, van Asperen PP, Selvadurai H, Fitzgerald DA. Normal development of the lung and premature birth. Paediatr Respir Rev 2010;11:135-42.

35. Battisti O. Lung compliance and airways resistance in healthy neonates. Pediatr Ther 2012;2:4.

36. Hooper SB, Fouras A, Siew ML, et al. Expired CO2 levels indicate degree of lung aeration at birth. PLoS ONE 2013;8:e70895.

37. Frerichs I, Amato MB, van Kaam AH, et al. Chest electrical impedance tomography examination, data analysis, terminology, clinical use and recommendations: consensus statement of the TRanslational EIT developmeNt stuDy group. Thorax 2017;72:83-93.

38. Miedema M, Waldmann A, McCall KE, Böhm SH, van Kamm AH, Tingay DG. Individualized multiplaner electrical impedance tomography in infants to optimize lung monitoring. Am J Respir Crit Care Med 2017;195:536-8.

39. Gattinoni L, Pelosi P, Crotti S, Valenza F. Effects of positive end-expiratory pressure on regional distribution of tidal volume and recruitment in adult respiratory distress syndrome. Am J Respir Crit Care Med 1995;151:1807-4.

40. Polglase GR, Hillman NH, Pillow JJ, et al. Positive end-expiratory pressure and tidal volume during initial ventilation of preterm lambs. Pediatr Res 2008;64:517-22. 